\title{
Mammalian Eps15 homology domain 1 potentiates angiogenesis of non-small cell lung cancer by regulating $\beta 2 A R$ signaling
}

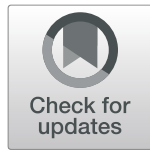

Ting Wang ${ }^{1 \dagger}$, Ying Xing ${ }^{1 \dagger}$, Qingwei Meng², Hailing Lu', Wei Liu', Shi Yan', Yang Song ${ }^{3}$, Xinyuan Xu', Jian Huang , Yue Cui ${ }^{1}$, Dexin Jia $^{1}$ and Li Cai ${ }^{*}$

\begin{abstract}
Background: Non-small cell lung cancer (NSCLC) is a devastating disease with a heterogeneous prognosis, and the molecular mechanisms underlying tumor progression remain elusive. Mammalian Eps15 homology domain 1 (EHD1) plays a promotive role in tumor progression, but its role in cancer angiogenesis remains unknown. This study thus explored the role of EHD1 in angiogenesis in NSCLC.

Methods: The changes in angiogenesis were evaluated through human umbilical vein endothelial cell (HUVEC) proliferation, migration and tube formation assays. The impact of EHD1 on $\beta 2$-adrenoceptor ( $\beta 2 A R$ ) signaling was evaluated by Western blotting, quantitative real-time polymerase chain reaction ( $q R T-P C R$ ) analysis, and enzymelinked immunosorbent assay (ELISA). The interaction between EHD1 and $\beta 2 A R$ was confirmed by immunofluorescence (IF) and coimmunoprecipitation (Co-IP) experiments, and confocal microscopy immunofluorescence studies revealed that $\beta 2 A R$ colocalized with the recycling endosome marker Rab11, which indicated $\beta 2 A R$ endocytosis. Xenograft tumor models were used to investigate the role of EHD1 in NSCLC tumor growth.
\end{abstract}

Results: The microarray analysis revealed that EHD1 was significantly correlated with tumor angiogenesis, and lossand gain-of-function experiments demonstrated that EHD1 potentiates HUVEC proliferation, migration and tube formation. EHD1 knockdown inhibited $\beta 2 A R$ signaling activity, and EHD1 upregulation promoted vascular endothelial growth factor A (VEGFA) and $\beta 2 A R$ expression. Interestingly, EHD1 interacted with $\beta 2 A R$ and played a novel and critical role in $\beta 2 A R$ endocytic recycling to prevent receptor degradation. Aberrant VEGFA or $\beta 2 A R$ expression significantly affected EHD1-mediated tumor angiogenesis. The proangiogenic role of EHD1 was confirmed in xenograft tumor models, and immunohistochemistry (IHC) analysis confirmed that EHD1 expression was positively correlated with VEGFA expression, microvessel density (MVD) and $\beta 2 A R$ expression in patient specimens.

Conclusion: Collectively, the data obtained in this study suggest that EHD1 plays a critical role in NSCLC angiogenesis via $\beta 2 A R$ signaling and highlight a potential target for antiangiogenic therapy.

Keywords: EHD1, NSCLC, Angiogenesis, $\beta 2 A R$ signaling, Endocytosis

\footnotetext{
*Correspondence: caili@ems.hrbmu.edu.cn

${ }^{\dagger}$ Ting Wang and Ying Xing are authors contributed equally to this work and

should be regarded as joint first authors.

${ }^{1}$ The Fourth Department of Medical Oncology, Harbin Medical University

Cancer Hospital, 150 Haping Road, Harbin 150040, China

Full list of author information is available at the end of the article
}

(c) The Author(s). 2019 Open Access This article is distributed under the terms of the Creative Commons Attribution 4.0 International License (http://creativecommons.org/licenses/by/4.0/), which permits unrestricted use, distribution, and reproduction in any medium, provided you give appropriate credit to the original author(s) and the source, provide a link to the Creative Commons license, and indicate if changes were made. The Creative Commons Public Domain Dedication waiver (http://creativecommons.org/publicdomain/zero/1.0/) applies to the data made available in this article, unless otherwise stated. 


\section{Background}

Lung cancer is the leading type of cancer worldwide [1], and non-small cell lung cancer (NSCLC) is the most frequent type of lung cancer, accounting for $87 \%$ of lung cancer cases, and has a 5-year survival rate of less than $17 \%$ [2]. Angiogenesis, the process of new blood vessel formation, is crucial for tumor growth, but the molecular mechanisms of angiogenesis in NSCLC remain unclear $[3,4]$. To improve patient outcome, new genes related to angiogenesis must be identified, and the molecular mechanisms underlying tumor neovascularization must be elucidated.

C-terminal Eps15-homology (EH) domain-containing protein (EHD1) regulates cellular receptor recycling from the endocytic recycling compartment to the plasma membrane $[5,6]$. Structurally, EHD1 has a single EH domain at its $\mathrm{C}$ terminus, a central coiled-coil region involved in oligomerization, and an $\mathrm{N}$-terminal regulatory region that binds to nucleotides [6, 7]. Importantly, C-terminal EHD proteins play an important role in regulating the transport of receptors, such as epithelial growth factor receptor [8], insulin-like growth factor receptor [9] and colony-stimulating factor-1 receptor [10]. An accumulating body of evidence implicates EHD1 in the development and progression of multiple types of cancers, such as breast cancer [8], thyroid cancer [11], ovarian cancer [12] and lung cancer [13]. Over the past five years, we have revealed that EHD1 overexpression in NSCLC predicts poor prognosis for patients and that EHD1 might play a pivotal role in tumor metastasis, stemness, chemotherapy resistance and epidermal growth factor receptor (EGFR)-tyrosine kinase inhibitor (TKI) resistance [13-15]. However, the involvement of EHD1 in tumor angiogenesis is unknown.

$\beta 2$-adrenoceptors ( $\beta 2 \mathrm{ARs}$ ), the best characterized $\beta$-adrenergic receptor proteins thus far, are prototypic and ubiquitous cell-surface proteins known as $\mathrm{G}$ proteincoupled receptors or seven-transmembrane receptors [16]. In cardiac disease, the activation of cardiomyocyte $\beta 2 A R$ by catecholamines leads to pathological responses [17]. Recently, focus has turned toward understanding the regulatory role of $\beta 2 A R$ in tumourigenesis [18]. Studies have shown that $\beta 2 \mathrm{AR}$ signaling stimulates pathological angiogenesis, which is an essential strategy used by tumor cells to obtain various nutrients and favors tumor growth and progression [19, 20]. Mechanistically, ligand- $\beta 2 A R-c A M P$-protein kinase A (PKA)-driven angiogenic growth factors are produced by endothelial cells [19]. Among these factors, vascular endothelial growth factors (VEGFs), particularly VEGFA, act as potent endothelial mitogens to induce a rapid and complete angiogenic response and organize vascular patterns [21]. $\beta 2 \mathrm{AR}$ signaling plays a well-documented role in promoting cancer progression in various malignancies, such as lung cancer [18], breast cancer [20] and gastric cancer
[22]. Therefore, further investigations are needed to better elucidate the $\beta 2 \mathrm{AR}$ signaling pathway and identify its upstream signal targets.

In this study, we examined the effect of EHD1 on tumor angiogenesis in NSCLC and found that EHD1 induces NSCLC angiogenesis by upregulating VEGFA expression. Furthermore, we demonstrated that EHD1 has a proangiogenic function through its regulation of the $\beta 2 \mathrm{AR}$ signaling pathway in NSCLC and revealed that EHD1 participates in $\beta 2 \mathrm{AR}$ endocytosis and recycling.

\section{Materials and methods \\ Microarray processing and analysis}

Detailed information on the microarray processing and analysis was described previously [13].

\section{Cell culture}

The human NSCLC cell lines NCI-H1650, PC9, NCIH1299, NCI-H827, NCI-H520, A549, NCI-H1975, PC14, NCI-H466, NCI-H2170 and NCI-H460 and human umbilical vein endothelial cells (HUVECs) were purchased from the American Type Culture Collection (ATCC, Manassas, VA, USA). The human NSCLC cell lines NCI-H1650, NCI-H1299, NCI-H827, NCI-H520, A549, NCI-H1975, PC14, NCI-H466, NCI-H2170 and NCI-H460 were maintained in 1640 medium supplemented with $10 \%$ fetal bovine serum (FBS, Gibco) and 1\% penicillin/streptomycin (Gibco). The PC9 cells were cultured in Dulbecco's modified Eagle's medium (DMEM) supplemented with 10\% FBS and $1 \%$ penicillin/streptomycin (Gibco). The HUVECs were incubated with Ham's F-12 K supplemented with $100 \mu \mathrm{g} / \mathrm{ml}$ heparin (Sigma), $50 \mu \mathrm{g} / \mathrm{ml}$ endothelial cell growth supplement (BD Biosciences), 10\% FBS (Gibco) and 1\% penicillin/ streptomycin (Gibco).

\section{Western blot analysis}

Antibodies against the following proteins were used in this study: EHD1 (ab109311, Abcam, Cambridge, MA, USA), VEGFA (ab1316, Abcam, Cambridge, MA, USA), $\beta 2 A R$ (ab182136, Abcam, Cambridge, MA, USA), $\beta 2 A R$ (sc-271,322, Santa Cruz), $\beta$-actin (TA-09, ZSGB-Bio, China) and glyceraldehyde-3-phosphate dehydrogenase (GAPDH; TA-08, ZSGB-Bio, China). The photodensity of Western blot bands was quantified using ImageJ software (U.S. National Institutes of Health, USA).

\section{Conditioned medium}

The A549 and NCI-H1650 cells were grown to 70-80\% confluence and then incubated in serum-free DMEM for $16 \mathrm{~h}$ or in serum-free DMEM with isoprenaline hydrochloride $(10 \mu \mathrm{m})$ (HY-B0468, MedChem Express) or ICI118,551 (ICI, $10 \mu \mathrm{m})$ (HY-13951, MedChem Express) for $16 \mathrm{~h}$ under the same conditions. Conditioned media 
(CMs) were collected, centrifuged at $2000 \mathrm{rpm}$ and $4{ }^{\circ} \mathrm{C}$ for $10 \mathrm{~min}$, filtered, and stored at $-70^{\circ} \mathrm{C}$.

\section{HUVEC proliferation assay}

HUVECs were seeded in 96-well plates at a density of 4000 cells per well and incubated with the corresponding CM, CM with VEGFA or CM with apatinib for $24 \mathrm{~h}$, $48 \mathrm{~h}$ and $72 \mathrm{~h}$. The cell viability rate was evaluated using the Cell Counting Kit-8 (Dojindo Molecular Technologies, Kumamoto, Japan). The optical density (OD) value was measured at $450 \mathrm{~nm}$.

\section{HUVEC migration assay}

HUVECs were grown to $70 \%$ confluence and serumstarved overnight. As previously described [23], $5 \times 10^{4}$ HUVECs were trypsinized, suspended in serum-free medium and seeded in the upper chamber insert (\#3422 Costar, Corning, NY, USA), and CM $(800 \mu \mathrm{l})$ was added to the lower chamber. After $24 \mathrm{~h}$, the migratory cells on the lower surface of the membrane were fixed with methanol and stained with crystal violet for $30 \mathrm{~min}$. The stained cells were observed and captured using a light microscope (Olympus), and the numbers of migratory cells in three random fields were quantified.

\section{HUVEC tube formation assay}

HUVECs $\left(8 \times 10^{4}\right)$ were starved overnight, incubated with the corresponding NSCLC CM $(200 \mu \mathrm{l})$ and seeded in a 24-well plate coated with Matrigel $(200 \mu \mathrm{l} /$ well, BD Biosciences). After $6 \mathrm{~h}$ of incubation at $37{ }^{\circ} \mathrm{C}$ with $5 \%$ $\mathrm{CO}_{2}$, the capillary tube structure was observed and captured using a light microscope (Olympus). The number of tubes was counted and compared between different groups [24]. Each condition was assessed in triplicate.

\section{The Cancer genome atlas (TCGA) and Cancer cell line encyclopedia (CCLE) data analysis}

Pan-cancer and lung cancer patient data were obtained from the TCGA database. Data on the expression of EHD1 and $\beta 2 A R$ in NSCLC cells were downloaded from the CCLE database.

\section{Enzyme-linked immunosorbent assay (ELISA)}

The VEGFA concentrations in the CMs were detected using a human VEGFA ELISA kit (USCN Life Science Inc., Wuhan, China). The measurements were performed in accordance to the instructions provided by the manufacturer and acquired with a microplate reader (BioTek, Winooski, VT, USA) at $450 \mathrm{~nm}$.

\section{qRT-PCR}

A qRT-PCR analysis was performed as previously described [25]. DNA was reacted with Fast SYBR Green Master Mix (Applied Biosystems) using the following primers: 5' -CCACAAGCTGGACATCTCCGATGAG-3' (forward) and 5'-GGGACCAGAAGGAGCCGATGTAGA C 3' (reverse) for EHD1; 5'-GATGGTGTGGAATTGTGT CAG-3' (forward) and 5'-GCAGGTCTCATTGGCATAG C-3' (reverse) for $32 A R$; 5 '-GAAGTGGTGAAGTTCATG GATGTCT-3' (forward) and 5' - ATGGTGATGTTGGA CTCCTCAGTG-3' (reverse) for VEGFA, and 5' -CTTAGT TGCGTTACACCCTTTCTTG-3' (forward) and 5'-CTGT CACCTTCACCGTTCCAGTTT-3' (reverse) for $\beta$-actin.

\section{Coculture experiments}

The A549 and NCI-H1650 cells were seeded into six-well coculture plates, grown to $70-80 \%$ confluence, and incubated in low-serum 1\% FBS-containing DMEM medium for $24 \mathrm{~h}$. A total of $5 \times 10^{4}$ HUVECs were seeded in each cell culture insert (pore size of $0.4 \mu \mathrm{m}, \mathrm{BD}$ Biosciences, CA, USA) for $24 \mathrm{~h}$. The HUVECs in these inserts were cocultured for $48 \mathrm{~h}$ with the pretreated A549 or NCI-H1650 cells, which were placed in the plate of the lower chamber, and then collected for Western blot analysis.

\section{Immunoprecipitation (IP) assay}

IP assays were conducted with the Crosslink Magnetic IP/ Co-IP kit (Thermo, Rockford, IL, USA). The measurements were performed based on the instructions provided by the manufacturer, as previously described [25].

\section{Immunofluorescence}

Measurements of immunofluorescence were performed as previously described [25]. The cells were incubated with the indicated primary antibodies against B2AR (1:100 dilution, sc-271,322, Santa Cruz) and Rab11 (1:100 dilution, \#5589, Cell Signaling Technology) overnight at $4{ }^{\circ} \mathrm{C}$ and then with Alexa Fluor 488-conjugated anti-mouse IgG (sc-516,140, Santa Cruz) or Alexa Fluor 647-conjugated anti-rabbit IgG (ab150075, Abcam, Cambridge, MA, USA) in the dark at room temperature for $1 \mathrm{~h}$. The stained cells were observed and captured using a laser-scanning confocal microscope (LSM510, Carl Zeiss, Inc.).

\section{Xenograft models}

Female, 4-5-week-old BALB/c nude mice were obtained from Beijing Vital River Laboratory Animal Technology Co., Ltd., and bred at the Animal Center of the Second Affiliated Hospital of Harbin Medical University. The $\mathrm{BALB} / \mathrm{c}$ nude mice were randomly divided into experimental groups ( $n=5 /$ group), and $5 \times 10^{6}$ cells were injected subcutaneously into the alar skin of the nude mice. Seven days after implantation, the mice were randomly divided into two subgroups. The initial luciferase signals and tumor volume were then measured, and the mice were then administered apatinib $(200 \mathrm{mg} / \mathrm{kg})$ or PBS once daily by oral gavage. Subsequently, the tumor 
volume was monitored with Vernier calipers every week for 4 weeks and calculated using the eq. $\left(\mathrm{L} \times \mathrm{W}^{2}\right) / 2$, where $\mathrm{L}$ is the length and $\mathrm{W}$ is the width. At day 28 , the initial luciferase signals were measured, the animals were sacrificed, and the tumor tissues were removed. Part of the tumor tissues was frozen at $-80^{\circ} \mathrm{C}$ for Western blot assay, and the remaining tissue was fixed and paraffin-embedded for immunohistochemical analysis.

\section{Immunohistochemistry (IHC)}

The detailed experimental immunohistochemical procedures were described previously [25]. The protein expression levels of EHD1, $\beta 2 A R$ and VEGFA were assessed by IHC with the corresponding anti-EHD1 (dilution 1:25, ab109311, Abcam, Cambridge, MA, USA), anti- $\beta 2$ AR (dilution 1:40, AF6117, Affinity Biosciences, China) and anti-VEGFA antibodies (dilution 1:100, ab 52,917, Abcam, Cambridge, MA, USA), respectively. Angiogenesis was evaluated through IHC staining of human and mouse tumor tissues with the anti-CD31 antibody (dilution 1:50, ab28364, Cambridge, MA, USA). The levels of EHD1, $\beta 2 A R$ and VEGFA staining were scored based on previously described criteria [25]. The microvessel density (MVD) in tumor samples was assessed based on CD31 staining. The MVD value was obtained as the median from the values obtained for three vascularized areas at $200 \times$ magnification. Using the average MVD in human tissues as the threshold, the tissues were split into a low group $(n \leq 9)$ and a high group based on their MVD status $(n>9)$.

\section{Statistical analysis}

All statistical analyses were performed with SPSS 22.0 and GraphPad Prism software. The data are expressed as the means \pm standard deviations (SDs). The differences between two groups were analyzed with Student's tests and the $\chi 2$ test. The survival analysis was performed using Kaplan-Meier analysis and log-rank tests. A two-tailed $p$ value of $<0.05$ was considered significant.

\section{Results}

\section{EHD1 expression predicts NSCLC and pan-cancer prognosis}

IHC analyses revealed that increased EHD1 expression was correlated with advanced $\mathrm{pT}$ classification and advanced pTNM stage in patients from Harbin Medical University Cancer Center (HMUCC) (Additional file 1: Table S1). We subsequently performed Kaplan-Meier analyses and found that high EHD1 expression predicts a poor prognosis in terms of both overall survival (OS) and disease-free survival (DFS) (Additional file 2: Figure S1a-b). Our results based on the TCGA database, which were mainly analyzed using the web-based tools in Gene Expression Profiling Interactive Analysis (GEPIA, http://gepia.cancer-pku.cn/) [26], showed that high EHD1 expression was an unfavorable predictor for NSCLC patients (Additional file 2: Figure S1c-d). Moreover, using data from 10,704 tumors in the TCGA database across 26 disease sites, we evaluated the predictive value of EHD1 gene expression for the prognosis of cancer patients. As shown in Additional file 2: Figure S1e and f, high EHD1 expression was a predictor of poor OS and progression-free interval in pan-cancer.

\section{EHD1 induces angiogenesis in NSCLC}

A microarray analysis performed using the Affymetrix $\mathrm{Hu}-$ man Gene 1.0 ST platform revealed a significant positive correlation between EHD1 and tumor angiogenesis and vascular endothelial cell proliferation and migration (Fig. 1a, Additional file 3: Table S2, Additional file 4: Table S3 and Additional file 5: Table S4). To test the effect of EHD1 on in vitro angiogenesis, A549 and NCI-H1650 cells were selected as a "loss-of-function" model due to their high expression of EHD1 [13]. We knocked down EHD1 expression in these NSCLC cells using shRNA targeting EHD1 (Fig. 1b). We then treated HUVECs with CM from untreated cells (UT), CM from control cells transfected with scrambled shRNA (Ctrl) or CM from EHD1downregulated cells (Sh). The evaluation of vitro angiogenesis activity based on the proliferation, migration and tube formation abilities of endothelial cells has been previously described [23]. Compared with the CMs from UT and Ctrl, the CM from Sh resulted in decreased HUVEC proliferation, migration and tube formation abilities (Fig. 1c-e).

To further validate the role of EHD1 in NSCLC angiogenesis, we conducted a rescue expression experiment in which Sh were transfected with a vector encoding the human EHD1 gene (the resulting cells were designated Sh/R) or with an empty vector (control, the resulting cells were designated Sh/Ctrl) (Additional file 6: Figure S2a). Treatment with the CM from Sh/R enhanced the abilities of HUVECs to proliferate, migrate and form tubes compared with treatment with the CMs from Sh and Ctrl (Additional file 6: Figure S2b-d).

\section{EHD1 promotes angiogenesis in a VEGFA-dependent manner}

VEGFA plays a critical role in angiogenesis [27]. The microarray data indicated that the mRNA levels of VEGFA were downregulated in the EHD1-knockdown NSCLC cells compared with the levels in the control cells (Fig. 2a, Additional file 3: Table S2, Additional file 4: Table S3 and Additional file 5: Table S4). Based on the TCGA database, we found a positive correlation between EHD1 and VEGFA expression $(p<0.0001, \mathrm{R}=0.14$; Fig. $2 \mathrm{~b})$. A Western blot assay using antibodies targeting VEGFA revealed that the EHD1-knockdown cells showed less VEGFA protein than the control cells (Fig. 2c). As 

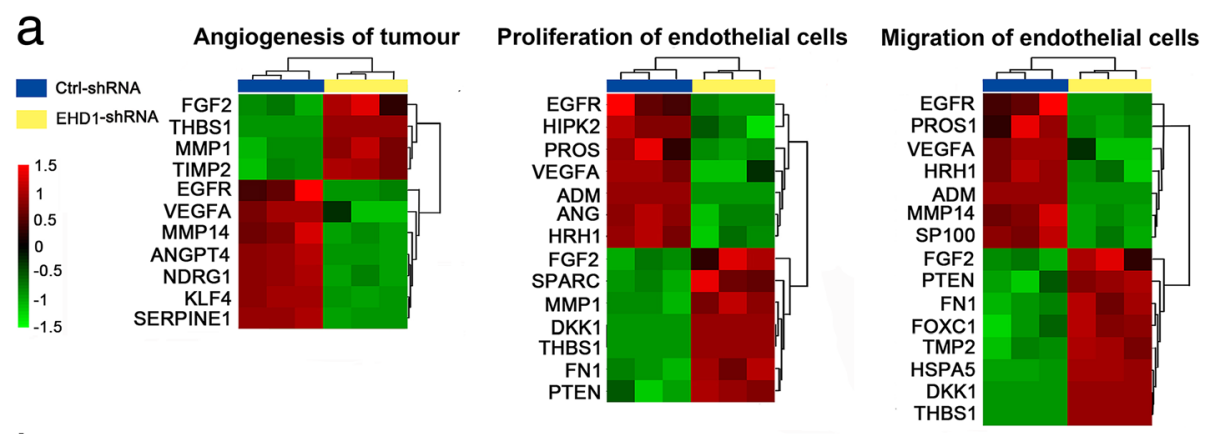

b

C
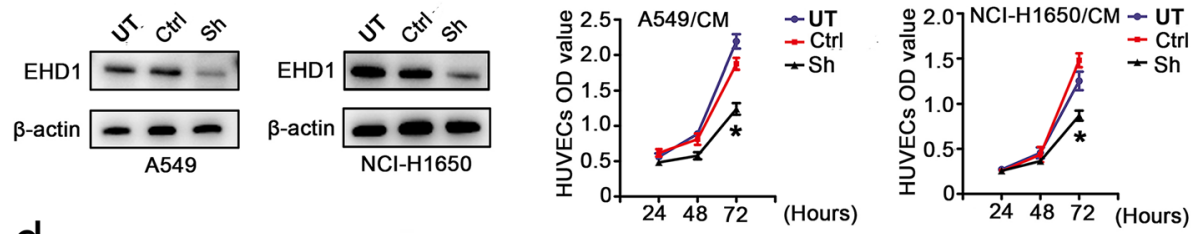

d $\quad$ A549/CM
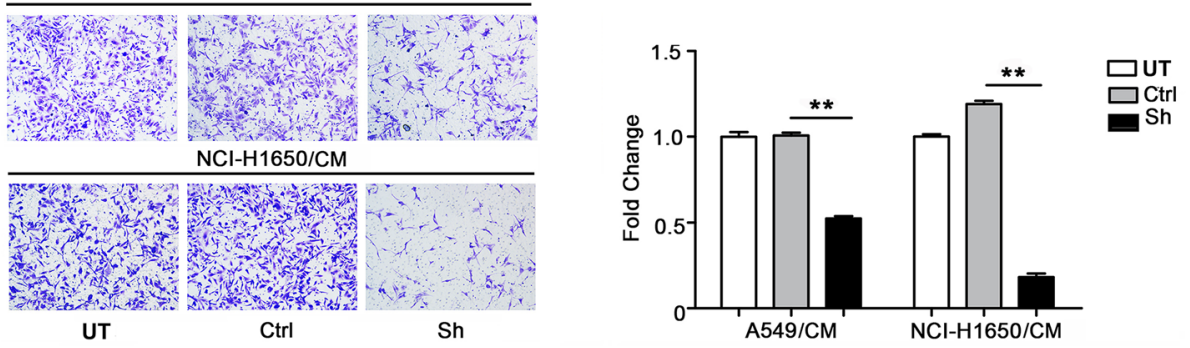

e

A549/CM

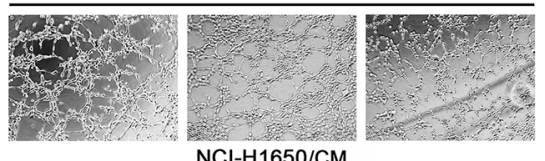

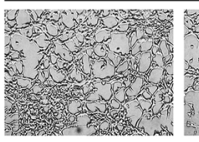

UT
$\mathrm{NCl}-\mathrm{H} 1650 / \mathrm{CM}$

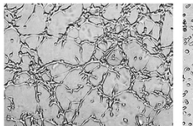

Ctrl

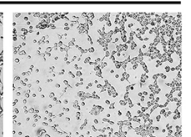

$\mathrm{Sh}$

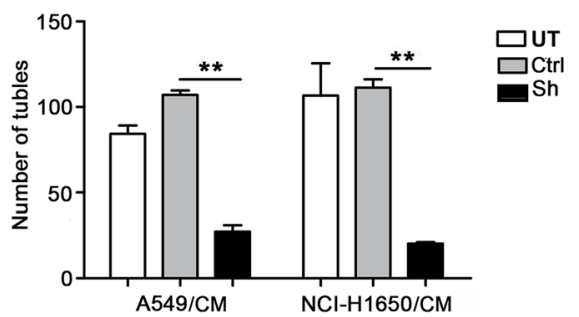

Fig. 1 EHD1 induces angiogenesis in NSCLC. a Microarray analysis demonstrating the positive correlation between EHD1 and tumour angiogenesis and vascular endothelial cell proliferation and migration. The pseudocolor represents the intensity scale for the EHD1 ShRNA vector versus the control, as calculated by log2 transformation. b Western blot analysis of EHD1 expression in A549 and NCI-H1650 cells after EHD1 knockdown. $\beta$-actin served as the loading control. c The viability of HUVECs was detected by CCK8 assay. HUVECs were incubated in 96-well plates with CMs from A549 and NCl-H1650 cells. d CMs were added to the lower chamber, and HUVECs were seeded on the upper chamber. After $24 \mathrm{~h}$ of incubation, HUVEC migration was assessed by counting the cells on the lower surface of the membrane; from left to right, the lanes show UT, Ctrl and ShEHD1. Scale bar, $100 \mu \mathrm{m}$. e HUVECs were incubated in 48-well plates with CMs from A549 and NCI-H1650 cells, and their tube formation was evaluated based on the number of tubes per field. ${ }^{*} p<0.05 ;{ }^{* *} p<0.01$

expected, the EHD1-knockdown cells showed less VEGFA mRNA expression than the control cells (Fig. 2d). As demonstrated by ELISA, the production of VEGFA was reduced in the NSCLC cells transfected with shRNA targeting EHD1 (Fig. 2e). To further investigate the involvement of VEGFA in EHD1-mediated angiogenesis in vitro, DMSO or VEGFA was added to the CM from Sh (Sh/CM), and subsequent in vitro HUVEC migration and tube formation assays revealed that the inhibitory effect of EHD1 knockdown on angiogenesis was reversed by VEGFA (Fig. 2f, g).

VEGFA promotes angiogenesis through activation of the phosphoinositide 3-kinase (PI3K)/Akt and Erk signaling pathways [28]. After coculture with Sh or Ctrl, we tested the expression of PI3K/Akt and Erk signaling molecules by Western blotting and demonstrated that p-AKT and p-Erk 


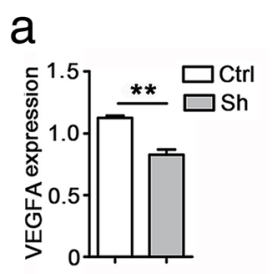

d
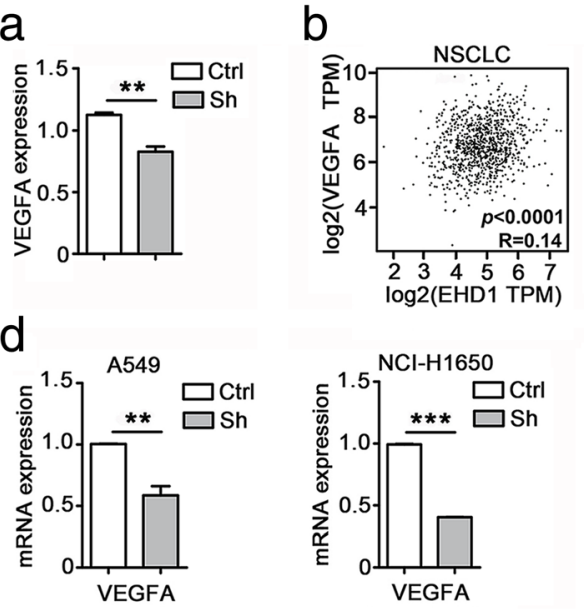

f

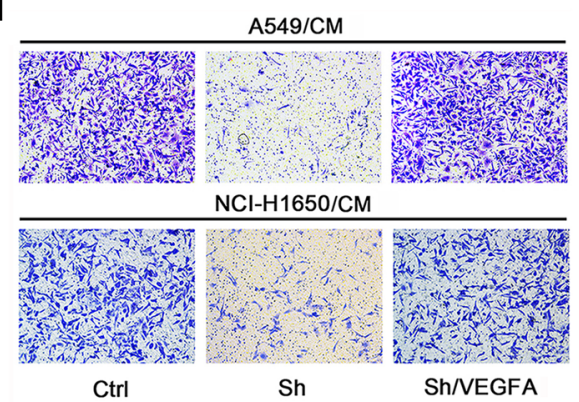

g

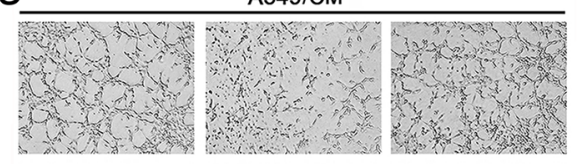
$\mathrm{NCl}-\mathrm{H} 1650 / \mathrm{CM}$

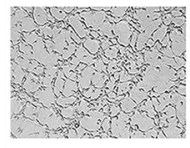

Ctrl

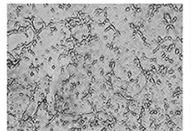

$\mathrm{Sh}$
C

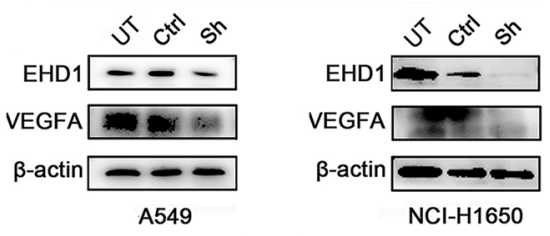

e

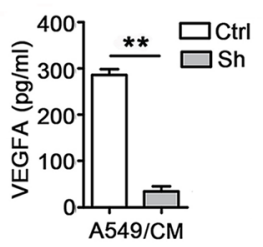

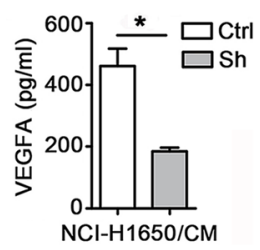

Fig. 2 EHD1-induced angiogenic effects depend on VEGFA expression. a mRNA expression of VEGFA. b Correlation between EHD1 and VEGFA expression in the TCGA database $(\mathrm{R}=0.14, p<0.0001)$. c Western blot analysis of VEGFA expression in A549 and NCI-H1650 cells after EHD1 knockdown. $\mathbf{d}$ qRT-PCR analysis of VEGFA mRNA levels in A549 cells and NCl-H1650 cells. e A549 and NCl-H1650 cells were incubated overnight with serum-free 1640 medium, and their CMs were used to detect the level of VEGFA secretion; the lanes in the left and right show Ctrl and Sh, respectively. $\mathbf{f}$ CMs were added to the lower chamber, and HUVECs were seeded on the upper chamber. After $24 \mathrm{~h}$ of incubation, HUVEC migration was assessed by counting the cells on the lower surface of the membrane. Scale bar, $100 \mu \mathrm{m}$. $\mathbf{g}$ HUVECs were incubated in 48-well plates with CMs from A549 and NCl-H1650 cells, and their tube formation abilities were evaluated based on the number of tubes per field

expression was significantly attenuated in HUVECs cocultured with Sh compared with their levels in HUVECs cocultured with Ctrl (Additional file 7: Figure S3).

Consistent with the above results, the reexpression of EHD1 in Sh increased the expression of VEGFA protein (Additional file 8: Figure S4a-b). Apatinib, a specific inhibitor of VEGFR2, completely abolished the EHD1-induced angiogenic effects (Additional file 8: Figure S4c, d). Together, these data show that the stimulation of cancer cell angiogenesis by EHD1 is highly dependent on VEGFA.
EHD1 activates $\beta 2 A R$ signaling in NSCLC

To understand the underlying mechanisms and identify the pathways driven by EHD1 in tumor angiogenesis, we analyzed microarray data using classical pathway enrichment analysis. The knockdown of EHD1 inhibited the $\beta$-adrenergic signaling pathway, which is involved in tumor angiogenesis and VEGFA regulation (Fig. 3a, Additional file 9: Table S5). More specifically, signaling through $\beta 2 A R$ has been shown to play a key role in the control of angiogenesis [20]. According to the data 
a

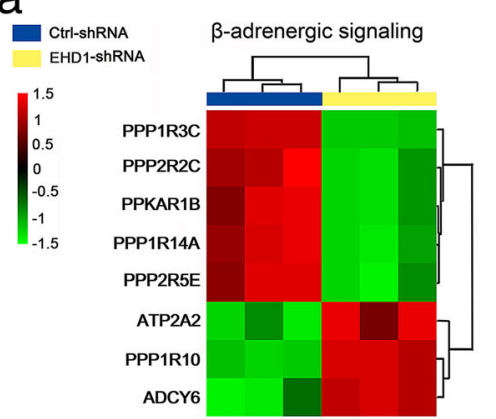

C
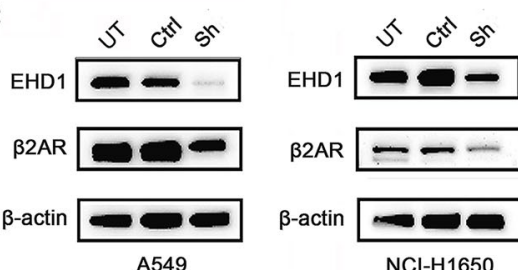

$\beta$-actin

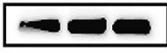

$\mathrm{NCl}-\mathrm{H} 1650$ b

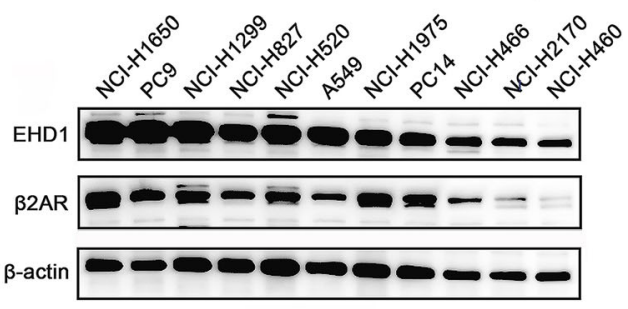

d

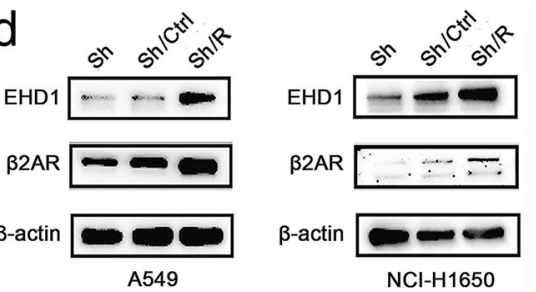

e

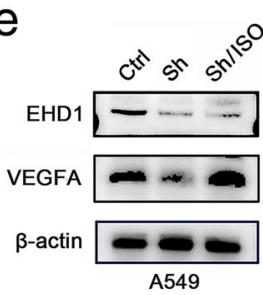

f
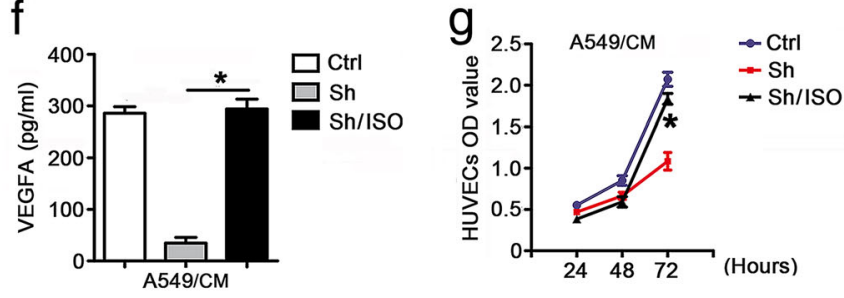

h

A549/CM

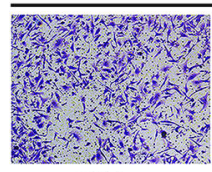

Ctrl

i

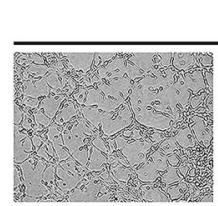

Ctrl

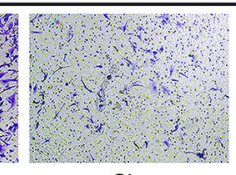

$\mathrm{Sh}$

A549/CM

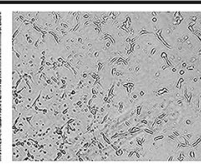

$\mathrm{Sh}$

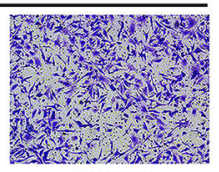

$\mathrm{Sh} / \mathrm{ISO}$

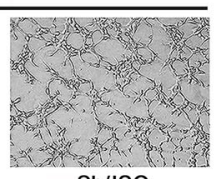

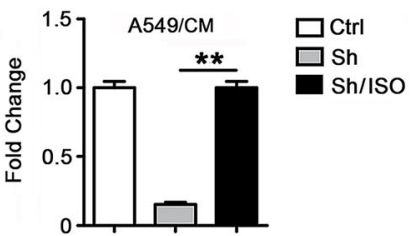

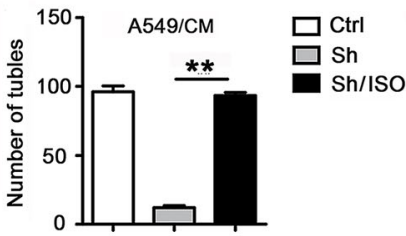

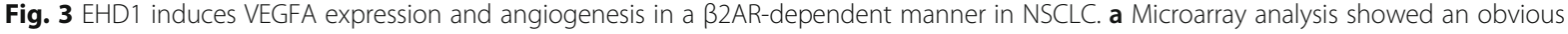

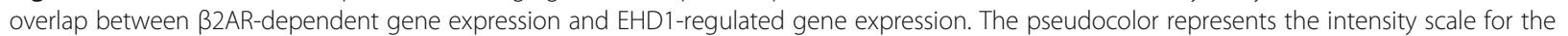
EHD1 ShRNA vector versus the control, as calculated by log2 transformation. b Western blot of EHD1 expression in human lung cancer cells. $\mathbf{c}$ Western blot analysis of $\beta 2 \mathrm{AR}$ expression in A549 and $\mathrm{NCl}-\mathrm{H} 1650$ cells after EHD1 knockdown. d Immunoblotting analysis of $\beta 2 \mathrm{AR}$ expression in A549 and NCl-H1650 cells after EHD1 reexpression. e A549 cells were transfected with vector or ShEHD1 and then treated with DMSO or ISO, and the EHD1 and VEGFA protein levels were assessed by immunoblotting. f A549 cells were incubated overnight with serum-free 1640 medium and corresponding reagents, and their CMs were used to test the level of VEGFA secretion by ELISA. $\mathbf{g}$ The viability of HUVECS was detected by CCK8 assay. HUVECs in 96-well plates were incubated with CMs from A549 cells. $\mathbf{h}$ CMs were added to the lower chamber, and HUVECs were seeded on the upper chamber. After $24 \mathrm{~h}$ of incubation, HUVEC migration was assessed by counting the cells on the lower surface of the membrane; from left to right, the lanes show Ctrl, Sh and Sh/ISO. Scale bar, 100 Mm. i HUVECs were incubated with CMs from A549 and NCI-H1650 cells in 48-well plates, and their tube formation abilities were evaluated based on the number of tubes per field

obtained from the TCGA database, EHD1 expression was positively correlated with $\beta 2 \mathrm{AR}$ expression (Additional file 10: Figure S5a) and the expression of the downstream gene hypoxia-inducible factor (HIF)- $1 \alpha$
(Additional file 10: Figure S5b) in lung squamous cell carcinomas and lung adenocarcinoma in the TCGA cohort. The correlation of EHD1 and $\beta 2 A R$ in NSCLC cell lines was also confirmed by Western blotting (Fig. 3b). 
These results prompted us to examine the effect of EHD1 on the regulation of $\beta 2 A R$ in NSCLC. A Western blotting analysis revealed that $\beta 2 \mathrm{AR}$ expression was significantly lower in EHD1-knockdown cells (Fig. 3c) but higher in EHD1-overexpressing cells (Fig. 3d) compared with the control cells. Interestingly, EHD1 knockdown significantly decreased $\beta 2 \mathrm{AR}$ protein but not mRNA expression (Additional file 11: Figure S6). These data clearly indicate that EHD1 regulates $\beta 2 \mathrm{AR}$ expression at the posttranscriptional level but not at the transcriptional level. Conversely, a $\beta 2 \mathrm{AR}$ agonist (isoproterenol, ISO) or a highly selective $\beta 2 A R$ antagonist (ICI118,551, ICI) did not alter EHD1 expression (Additional file 12: Figure S7). Taken together, the results indicate that EHD1 is a critical factor controlling $\beta 2 \mathrm{AR}$ expression, that $\beta 2 \mathrm{AR}$ has no impact on EHD1, and that EHD1 activates $\beta 2 A R$ signaling in NSCLC.

\section{EHD1 induces VEGFA expression and angiogenesis in a B2AR-dependent manner in NSCLC}

We subsequently tested whether $\beta 2 A R$ is necessary for EHD1-induced VEGFA expression and angiogenesis by treating Sh with ISO, an $\beta 2 \mathrm{AR}$ agonist. As shown in Fig. 3e and f, ISO reversed the decrease in VEGFA expression observed after EHD1 knockdown in NSCLC cells. To explore the role of $\beta 2 A R$ in EHD1-induced angiogenesis, HUVECs were incubated with Ctrl-CM, Sh-CM or Sh-CM+ISO, and the results showed that treatment with ISO rescued the $\mathrm{Sh} /$ CM-mediated decrease in HUVEC proliferation, migration and tube formation (Fig. 3g-i).

We subsequently used ICI, a highly selective $\beta 2 A R$ antagonist. As demonstrated by Western blotting and ELISA, the overexpression of EHD1 increased VEGFA expression, and ICI eliminated this effect (Additional file 13: Figure S8a, b). In vitro angiogenesis assays showed that the viability of HUVECs treated with Sh/R-CM + ICI was significantly lower than that of HUVECs treated with Sh/R-CM (Fig. 8c). Similar results were also obtained in the HUVEC migration and tube formation assays (Additional file 13: Figure S8d, e). These data suggest that $\beta 2 A R$ is critical for EHD1-induced VEGFA expression and is required for EHD1-stimulated angiogenesis.

\section{EHD1 knockdown results in impaired $\beta 2 A R$ endocytic recycling}

Our observations demonstrated that EHD1 positively regulates $\beta 2 A R$ protein expression but not mRNA expression (Additional file 11: Figure S6). Given that EHD1 plays a critical role in receptor endocytosis and recycling, we speculated that EHD1 regulates $\beta 2 A R$ protein via a recycling route through the endocytic pathway. To test this hypothesis, we validated the existence of an interaction between EHD1 and $\beta 2 \mathrm{AR}$. We first stained
NSCLC cells with anti-EHD1 and anti- $\beta 2 A R$ antibodies and observed the colocalization of EHD1 and $\beta 2 A R$ protein by confocal microscopy (Fig. 4a, b). The interaction between EHD1 and $\beta 2 A R$ protein was further confirmed by coimmunoprecipitation (Co-IP) assays, which demonstrated that endogenous EHD1 colocalized with $\beta 2 \mathrm{AR}$ (Fig. 4c) and that EHD1 was pulled down by endogenous B2AR (Fig. 4d).

Rab11, which is a recycling endosome marker, interacts with EHD1 through NPF-EH domain interactions and plays a role in recycling from the endocytic recycling compartment [29]. We thus subsequently investigated the colocalization of $\beta 2 \mathrm{AR}$ and Rab11. A confocal microscopy analysis revealed that the colocalization of B2AR and Rab11 in NSCLC cells was significantly decreased by EHD1 knockdown (Fig. 4e). In contrast, this decrease in $\beta 2 A R-R a b 11$ colocalization was reversed by EHD1 reexpression (Fig. 4f).

Once internalized, the receptors are either recycled back to the plasma membrane or sent to late endosomes and on to the lysosomal pathway for degradation (signal termination) [30]. Cycloheximide chase experiments were performed to analyze the role of EHD1 in $\beta 2 A R$ stabilization. The half-life of $\beta 2 A R$ in EHD1-knockdown cells was markedly shorter than that in control cells, which suggested that in the absence of EHD1, lysosomal delivery is dominant, resulting in $\beta 2 A R$ protein degradation (Fig. 4g, h). Altogether, these data suggest that EHD1 enhances the endocytic recycling of $\beta 2 A R$ and inhibits the degradation of this receptor.

\section{EHD1 promotes tumor growth and angiogenesis in vivo}

To further investigate the role of EHD1 in NSCLC angiogenesis in vivo, we established xenograft models using nonobese diabetic (NOD)-severe combined immunodeficient (SCID) mice. A549 cells stably transfected with EHD1-Ctrl (Ctrl), EHD1-shRNA (Sh), EHD1-shRNA/Ctrl (Sh/Ctrl) and EHD1-shRNA/R (Sh/R) were subcutaneously injected into the alar skin of the mice, and the tumor growth over 7 and 28 days after implantation was monitored. The mice were sacrificed 28 days after implantation, and the tumors were removed for further analysis.

Luciferin-based bioluminescence imaging at 7 and 28 days revealed that the knockdown of EHD1 significantly decreased the tumor development potential of A549 cells (Fig. 5a). Relative to the those implanted with the control cells, the mice implanted with EHD1-knockdown cells showed significantly less tumor growth and a decreased tumor weight (Fig. 5b-c). IHC and immunohistofluorescence analyses demonstrated that the expression of CD31, a marker of the MVD, was lower in the tumor tissue obtained with the Sh than in that obtained with the Ctrl (Fig. 5d-e). Conversely, the tumors formed from the $\mathrm{Sh} / \mathrm{R}$ showed higher luciferase activity (Fig. $5 \mathrm{f}$ ), larger 


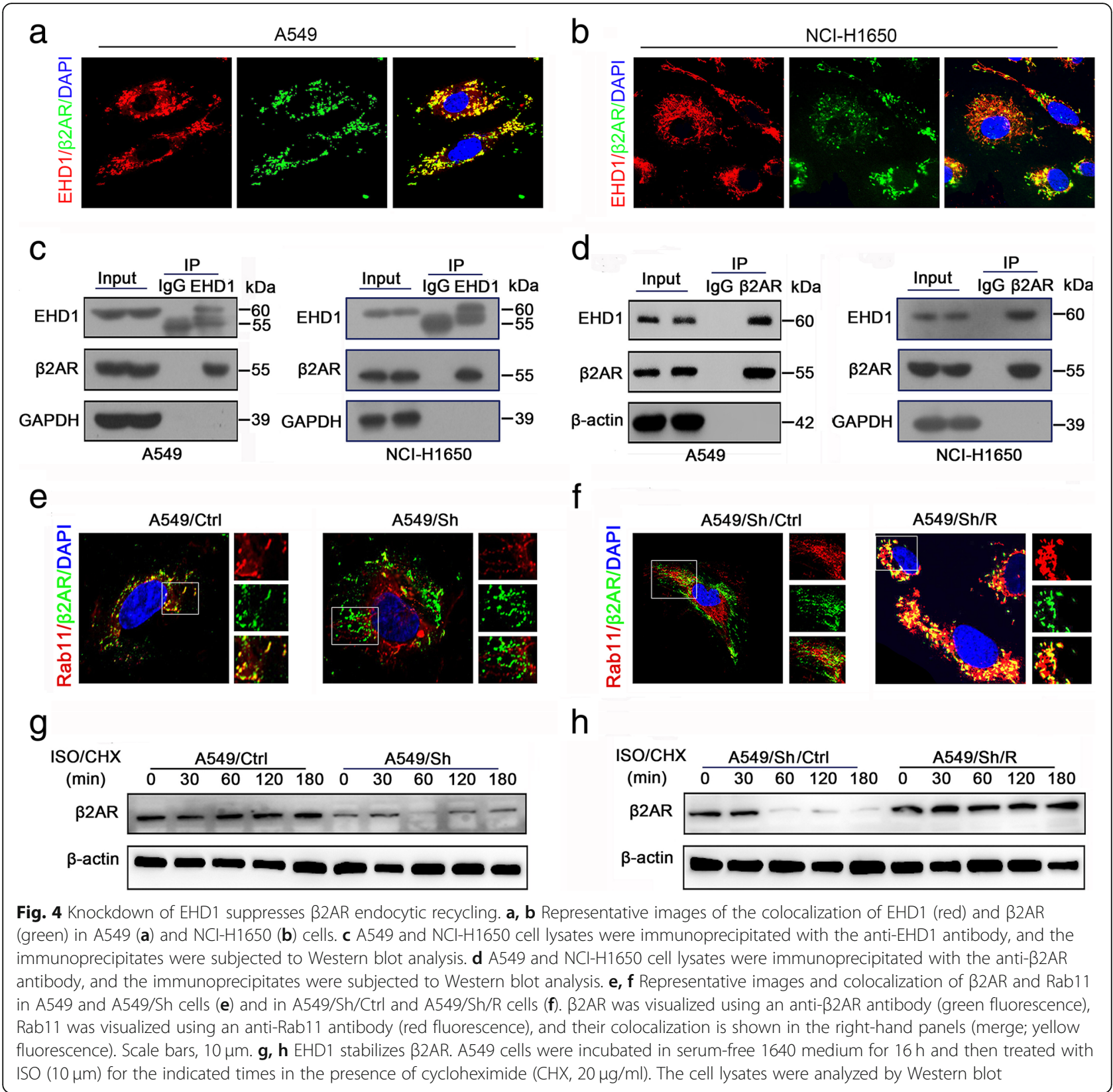

volumes (Fig. 5g) and heavier weight (Fig. 5h) than those obtained from the Sh/Ctrl. In addition, increased CD31 staining was detected in the tumor tissue obtained from Sh/R compared with that obtained from Sh/Ctrl (Fig. 5i, j). Taken together, these data demonstrate that EHD1 plays an important role in promoting angiogenesis and tumor growth.

\section{Targeting VEGFA represses EHD1-induced tumor growth and angiogenesis in vivo}

In addition, we investigated the impact of $\beta 2 A R$ signaling on EHD1-induced NSCLC angiogenesis and tumor growth in vivo. We detected the expression of EHD1,
B2AR and VEGFA in freshly frozen xenograft tumor tissue by Western blot and found that the EHD1 levels were positively correlated with $\beta 2 \mathrm{AR}$ and VEGFA expression (Fig. 6a, b). Furthermore, once the tumors reached a volume of $\sim 100 \mathrm{~mm}^{3}$, the mice transplanted with $\mathrm{Sh} / \mathrm{R}$ were randomly divided into two groups: 1 ) Sh/R-Ctrl and 2) Sh/R-apatinib. The Sh/R-apatinib mice showed a decreased tumor volume and a lower tumor weight than the control mice, which suggested that VEGFA inhibition impaired the EHD1-induced effect on tumor growth (Fig. 6c-e). Importantly, the IHF results confirmed the decreased expression of CD31 protein detected by IHC analysis in the xenograft tumor tissues of 

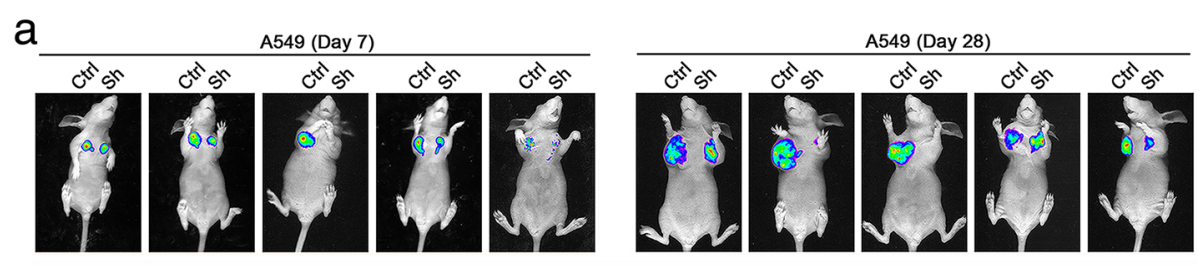

b
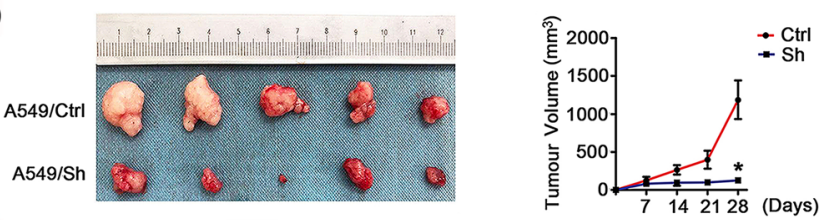

C

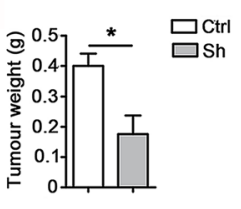

d
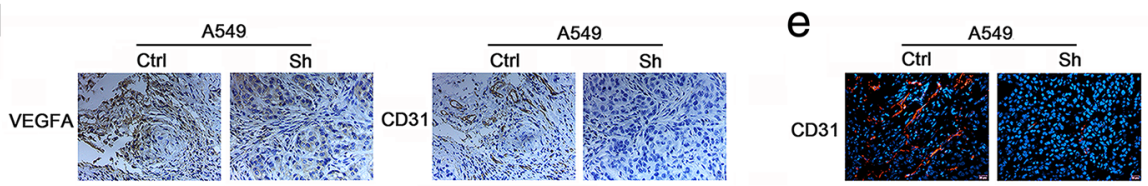

f
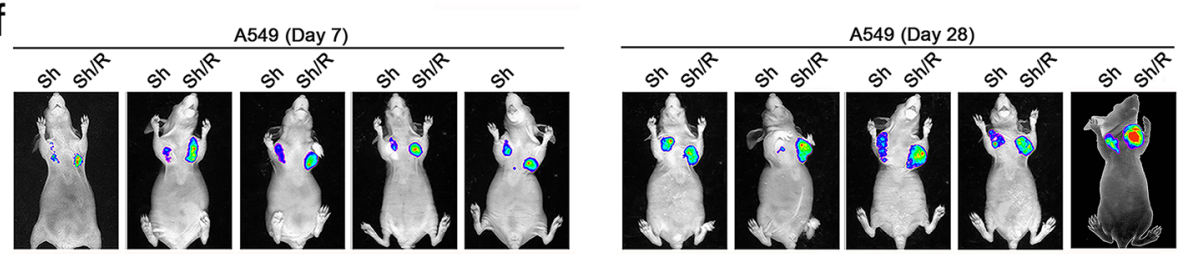

9
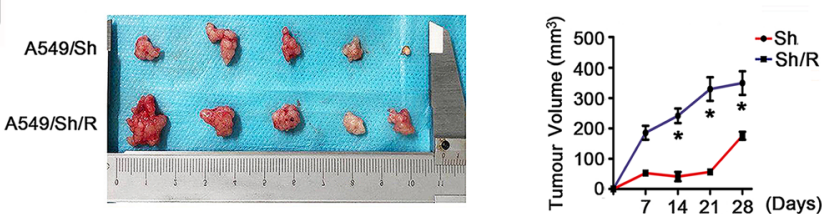

$\mathrm{h}$

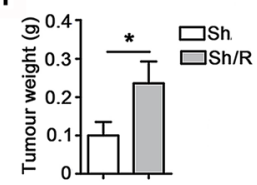

i
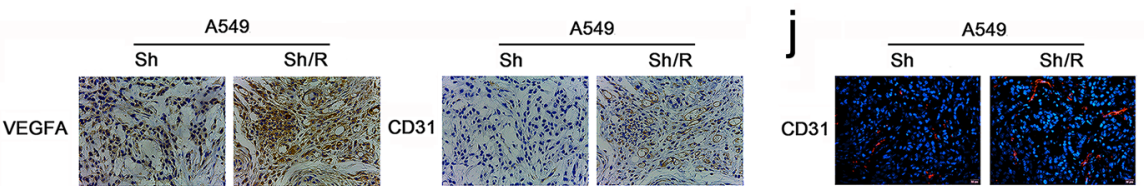

Fig. 5 EHD1 contributes to tumor growth and angiogenesis in vivo. a-e A549 luciferase cells stably expressing control ShRNA or EHD1 ShRNA were injected into the alar skin of nude mice, and images of the resulting tumors in the mice on days 7 and 28 are shown (a). A photograph of the tumors at day 28 post injection is shown (left), and the tumor growth curves of the mice on days 7 to 28 are presented (right) (b). The tumor weights were measured, and the data are presented as the means \pm SDs ( $n=5$, each) (c). Representative images of IHC staining for VEGFA and CD31 in xenograft tumor tissues are presented (d). Representative immunofluorescent staining of CD31 is shown (e). f-i A549-luciferase cells stably expressing EHD1 ShRNA or EHD1 ShRNA/R were injected into the alar skin of nude mice. Images of the resulting tumors in the mice on days 7 and 28 are shown (f). A photograph of the tumors at day 28 postinjection is shown (left), and the tumor growth curves of the mice on days 7 to 28 are presented (right) $(\mathbf{g})$. The tumor weights were measured, and the data are presented as the means \pm SDs $(n=5$, each) $(\mathbf{h})$. Representative images of $\mathrm{IHC}$ staining for VEGFA and CD31 in xenograft tumor tissues are presented (i). Representative immunofluorescent staining of CD31 is shown (j). ${ }^{*} p<0.05$

the Sh/R-apatinib group (Fig. 6f, g). These data suggest that targeting $\beta 2 A R$ signaling blocks EHD1-induced tumor growth and angiogenesis in vivo.

\section{EHD1, $\beta 2 A R$, VEGFA and CD31 are coordinately} overexpressed in clinical NSCLC specimens

To further examine the relationship between EHD1 and angiogenesis in human NSCLC, we performed IHC staining of EHD1, $32 A R$, VEGFA and CD31 in 96 NSCLC patient specimens. Consistent with our observations in tumor cell lines and xenograft models, the distribution and intensity of EHD1 were positively correlated with $\beta 2 \mathrm{AR}$, VEGFA and CD31 in NSCLC tissue specimens (Additional file 14: Figure S9a). EHD1 was highly expressed in $40.6 \%$ of NSCLC cases $(n=96)$. Moreover, the patients with high EHD1 expression also showed high $32 \mathrm{AR}$ 
a

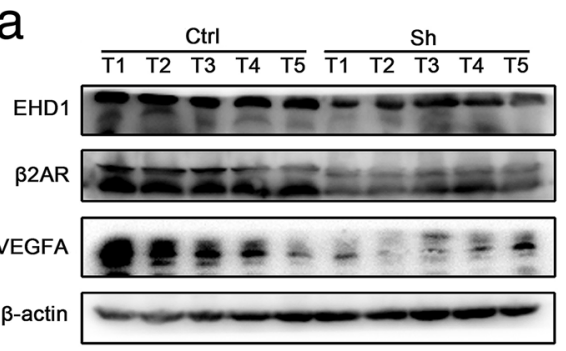

C

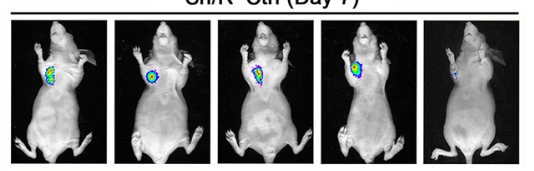

Sh/R-Apatinib (Day 7)

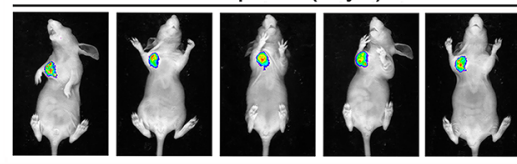

d
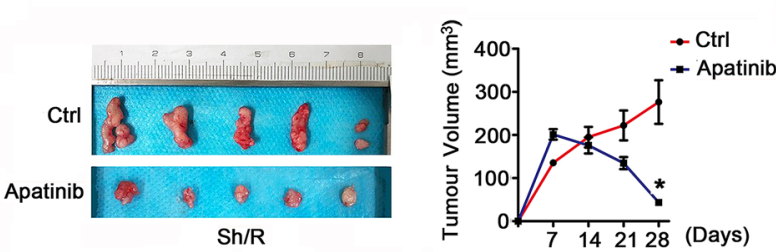

f
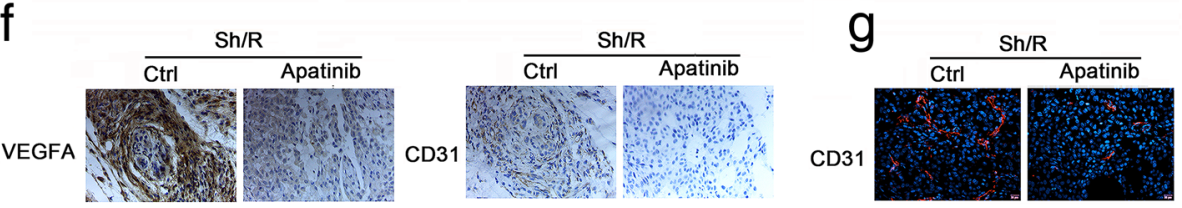
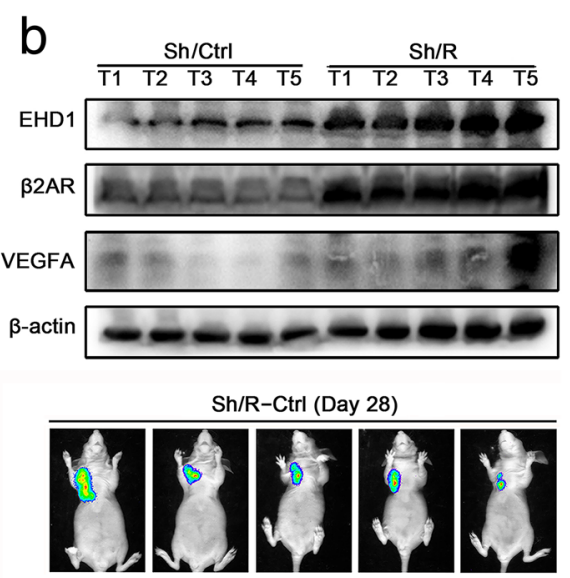

Sh/R-Apatinib (Day 28)

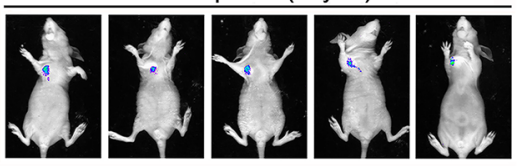

e

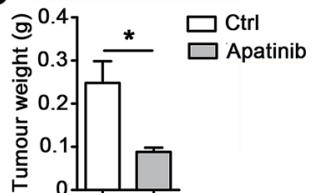

$\mathrm{h}$

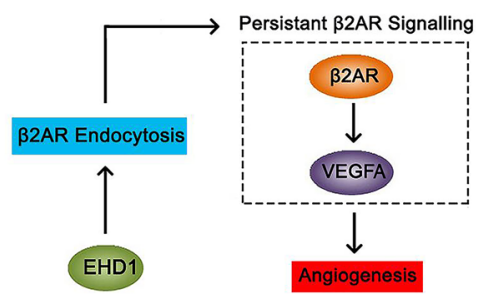

Fig. 6 Targeting of VEGFA represses EHD1-induced tumor growth and angiogenesis in vivo. a-b The expression of EHD1, $\beta 2 A R$ and VEGFA in freshly frozen xenograft tumor tissue was analyzed by Western blot. c-g A549 luciferase cells stably expressing EHD1 ShRNA/R-Ctrl or EHD1 ShRNA/R-apatinib were transplanted into the alar skin of nude mice. Images of the resulting tumors in the mice on days 7 and 28 are shown (c). A photograph of the tumors on day 28 is shown (left), and the tumor growth curves of the mice on days 7 to 28 are presented (right) (d). The tumor weights were measured, and the data are presented as the means \pm SDs ( $n=5$, each) (e). Representative images of IHC staining for VEGFA and CD31 in xenograft tumor tissues are presented (f). Representative immunofluorescent staining of CD31 is shown (g). $\mathbf{h}$ Model of EHD1induced $\beta 2$ ARNEGFA expression and angiogenesis in NSCLC

(Additional file 14: Figure S9b), VEGFA (Additional file 14: Figure S9c) and CD31 expression (Additional file 14: Figure S9d). The intratumoral MVD is used to examine the role of vascularization within the malignant process [31], and thus, MVD scores were calculated by counting the numbers of CD31-positive vessels in whole tumor cross-sections [32]. This analysis revealed that the EHD1 protein levels were positively correlated with the MVD scores (Additional file 14: Figure S9e), which clearly indicated that high EHD1 expression was associated with elevated angiogenesis in NSCLC patients.

\section{Discussion}

In the present study, we provide the first demonstration that EHD1 potentiates tumor angiogenesis in vitro and in vivo. Angiogenesis is considered a cancer progressive 
factor in tumor growth and metastasis, and antiangiogenic therapy is thought to be an effective therapeutic approach that achieves the expected outcome in patients with cancer [3, 33]. Our findings suggest that EHD1 represents a promising target for antiangiogenetic lung cancer treatment. We and other researchers have reported that EHD1 plays a significant tumor-promoting role as an oncogene in various cancers $[8,11]$. Our previous study revealed that EHD1 promotes lung cancer metastasis by inducing epithelial-mesenchymal transition [13]. Given the impact of angiogenesis in cancer metastasis, we will address whether angiogenesis is required for EHD1-induced cancer metastasis in the future.

Here, we confirmed that EHD1 induced VEGFA expression and increased VEGFA secretion. Indeed, VEGFA is the master mediator of tumor angiogenesis and stimulates the migration and proliferation of cultured endothelial cells in different cancer types [34, 35]. The angiogenic function of VEGFA is primarily achieved by binding to receptors, predominantly VEGFR2, on endothelial cells [27]. The binding of VEGF to VEGFR2 induces changes in a variety of downstream signaling pathways, particularly the PI3K/AKT, mitogen-activated protein kinase kinase $(\mathrm{MEK}) /$ Erk and P38-mitogen-activated protein kinase (MAPK) pathways, and thereby affects the biological function of endothelial cells [28]. In line with these studies, our results indicated that EHD1-induced VEGFA led to high PI3K/AKT and MEK/Erk signaling pathway activity in endothelial cells.

Moreover, we revealed that EHD1 promoted angiogenesis and tumor growth in a VEGFA-dependent manner. Agents that selectively target VEGFA, its receptor or its downstream signaling pathway effectively improve the survival rates of patients with a variety of cancers [33]. Ovarian cancer patients with EHD1 overexpression exhibit significantly worse responses to bevacizumab, which targets VEGFA [12]. Therefore, we need a large number of NSCLC patients treated with apatinib to analyze the association between the expression of EHD1 and the clinical prognosis of apatinib-treated patients and to further clarify the guiding role of EHD1 in clinical antiangiogenic therapy. In addition, our results will help improve strategies for the selection of NSCLC patients who may particularly benefit from agents that selectively target the VEGFA pathway.

Our current study showed that EHD1 potentiates angiogenesis and tumor growth via the $\beta 2 A R$ signaling pathway. A large body of evidence suggests that $\beta 2 A R$ signaling activation upregulates the expression of VEGFA and promotes angiogenesis and tumor growth [20, 22]. In our study, although the TCGA data showed that EHD1 was positively correlated with $\beta 2 A R$ mRNA expression in NSCLC, the in vitro experiment results showed that EHD1 did not positively regulate $\beta 2 \mathrm{AR}$ mRNA expression and that the aberrant $\beta 2 \mathrm{AR}$ signaling pathway did not regulate EHD1 expression. Thus, there might be a co-responsive relationship between EHD1 and $\beta 2 A R$ in which EHD1 and $\beta 2 A R$ respond to other molecular changes, such as the same transcription factor. In future work, we would like to explore the relationship between EHD1 and $\beta 2 \mathrm{AR}$ at the transcriptional level.

Moreover, we have indicated that EHD1 governs $\beta 2 A R$ signaling by regulating the recycling of $\beta 2 \mathrm{AR}$ from the endocytic recycling compartment to the plasma membrane. $\beta 2 A R$, as a seven-transmembrane $G$ protein-coupled receptor, undergoes internalization and is then transported to the endosome, from where it is either transported to the late endosome for degradation or transported to the recycling endosome for recovery and transport to the cell surface for persistent activation [30]. Endocytosis trafficking plays a key role in controlling the activity of $\beta 2 A R$ [36]. A large body of evidence from many investigators supports the notion that $\beta 2 A R$ plays a carcinogenic role dependent on receptor endocytosis [37].

EHD1 regulates the endocytic recycling of transmembrane receptors, such as epithelial growth factor receptor, insulin-like growth factor receptor and colony-stimulating factor-1 receptor [8-10]. Our data reveal a novel function of EHD1 as a regulator of $\beta 2 A R$ recycling and demonstrate a requirement for EHD1 in $\beta 2 A R$-mediated downstream functions. Despite its role in endocytic recycling, EHD1 also plays a role in the transport of newly synthesized receptors from the Golgi to the cell surface [10]. Future studies in our laboratory will test this function of EHD1.

Here, we propose a working model of EHD1 function in tumor angiogenesis. The coupling of $\beta 2 A R$ with its ligands induces the internalization of $\beta 2 \mathrm{AR}$, which is then delivered to early endosomes. EHD1 promotes the endocytic recycling of $\beta 2 \mathrm{AR}$, resulting in an increased amount of $\beta 2 A R$ available for oncogenic signaling. Thus, EHD1 overexpression leads to persistent $\beta 2 A R$ signaling activity, and upregulated VEGFA stimulates tumor angiogenesis (Fig. 6h).

\section{Conclusion}

Taken together, the results obtained in this study reveal that EHD1 potentiates NSCLC growth and angiogenesis through the $\beta 2 A R / V E G F A$ signaling pathway both in vitro and in vivo. Moreover, EHD1 governs $\beta 2 A R$ signaling by promoting the endocytic recycling of $\beta 2 \mathrm{AR}$. These observations improve our understanding of how EHD1 impacts cancer development and progression and provide new insights into the underlying mechanisms of NSCLC angiogenesis, which emphasize that EHD1 is a potential antiangiogenic therapeutic target in NSCLC. 


\section{Additional files}

Additional file 1: Table S1. Association between EHD1 expression and clinicopathological characteristics of NSCLC patients. (DOC $63 \mathrm{~kb}$ )

Additional file 2: Figure S1. EHD1 overexpression is associated with poor prognosis. a, b Kaplan-Meier curves of overall survival (a) and disease-free survival (b) for patients with NSCLC from Harbin Medical University Cancer Center (HMUCC). c, d Kaplan-Meier curves for overall survival (c) and disease-free survival (d) for patients with NSCLC in the TCGA database. e Kaplan-Meier overall survival curves for cancer patients with low EHD1 expression $(n=5037)$ and high EHD1 expression ( $n=5039 ; p=0.0018)$. $f$ Kaplan-Meier progression-free interval curves for cancer patients with low $(n=5118)$ and high $(n=5117)$ expression of EHD1 ( $p<0.0001)$. (TIF $4499 \mathrm{~kb})$

Additional file 3: The association between EHD1 and tumor angiogenesis. (XLSX $59 \mathrm{~kb}$ )

Additional file 4: The association between EHD1 and vascular endothelial cell proliferation. (XLSX $25 \mathrm{~kb}$ )

Additional file 5: The association between EHD1 and vascular endothelial cell migration. (XLSX $26 \mathrm{~kb}$ )

Additional file 6: Figure S2. Reexpression of EHD1 promotes angiogenesis. a Western blot analysis of EHD1 expression in A549 and $\mathrm{NCl}-\mathrm{H} 1650$ cells after EHD1 reexpression. b The viability of HUVECs was detected by CCK8 assay. HUVECs were incubated in 96-well plates with CMs from A549 and NCl-H1650 cells. c CMs were added to the lower chamber, and HUVECs were seeded on the upper chamber. After $24 \mathrm{~h}$ of incubation, HUVEC migration was assessed by counting the cells on the lower surface of the membrane; from left to right, the lanes show Sh/UT, Sh/Ctrl and Sh/R. Scale bar, $100 \mu \mathrm{m}$. d HUVECs were incubated in 48-well plates with CMs from A549 and $\mathrm{NCl}-\mathrm{H} 1650$ cells, and their tube formation abilities were evaluated based on the number of tubes per field. ${ }^{*} p<0.05 ;{ }^{* *} p<0.01$. (TIF $10271 \mathrm{~kb}$ )

Additional file 7: Figure S3. EHD1 knockdown attenuates p-AKT and p-Erk expression in HUVECs. p-AKT and p-Erk expression in HUVECs cocultured with Sh was significantly attenuated compared with that in HUVECs cocultured with Ctrl. (TIF 7820 kb)

Additional file 8: Figure S4. Reexpression of EHD1 increases VEGFA expression and angiogenesis. a Western blot analysis of VEGFA expression in $\mathrm{A} 549$ and $\mathrm{NCl}-\mathrm{H} 1650$ cells after EHD1 reexpression. $\beta$-actin served as the loading control. b A549 and NCl-H1650 cells were incubated overnight with serum-free1640 medium, and their CMs were used to detect the level of VEGFA secretion; from left to right, the lanes show Sh/Ctrl and Sh/R, respectively. c CMs were added to the lower chamber, and HUVECs were seeded on the upper chamber. After $24 \mathrm{~h}$ of incubation, HUVEC migration was assessed by counting the cells on the lower surface of the membrane. Scale bar, $100 \mu \mathrm{m}$. d HUVECs were incubated in 48well plates with CMs from A549 and $\mathrm{NCl}-\mathrm{H} 1650$ cells, and their tube formation abilities were evaluated based on the number of tubes per field. ${ }^{*} p<0.05 ;{ }^{* *} p<0.01$. (TIF $10245 \mathrm{~kb}$ )

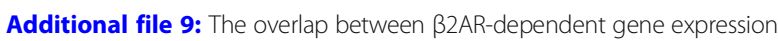
and EHD1-regulated gene expression. (XLSX $14 \mathrm{~kb}$ )

Additional file 10: Figure S5. EHD1 expression is positively correlated with $\beta 2$ AR signaling in NSCLC. a Analyses of TCGA lung adenocarcinoma and lung squamous cell carcinoma samples show that EHD1 expression is positively correlated with $\beta 2 A R$ expression. $b$ Analyses of TCGA lung adenocarcinoma and lung squamous cell carcinoma samples show that EHD1 expression is positively correlated with HIF1-a expression. (TIF 8835 kb)

Additional file 11: Figure S6. EHD1 has no effect on $\beta 2 A R$ mRNA expression. qRT-PCR analysis of EHD1 and $\beta 2 A R$ mRNA levels in A549 cells; the lanes show Ctrl and Sh. (TIF $880 \mathrm{~kb}$ )

Additional file 12: Figure S7. EHD1 expression is not affected by activation or inhibition of the $\beta 2 A R$ signaling pathway. Western blot analysis of EHD1 expression in A549 cells after EHD1 knockdown and reexpression. A549/Sh cells were treated with ISO for $4 \mathrm{~h}$ to activate $\beta 2 \mathrm{AR}$ signaling, and A549/Sh/R cells were treated with $\mathrm{ICl}$ for $4 \mathrm{~h}$ to inhibit B2AR signaling activation. (JPG $1514 \mathrm{~kb}$ )
Additional file 13: Figure S8. Blocking $\beta 2 A R$ inhibited EHD1-induced VEGFA expression and angiogenesis. a Western blot analysis of VEGFA expression in A549 cells after EHD1 knockdown and reeexpression; the lanes show Sh/Ctrl, Sh/R, and Sh/R/ICl, respectively. b A549 cells were incubated overnight with serum-free 1640 medium and corresponding reagents, and their CMs were used to detect the level of VEGFA secretion. c The viability of HUVECs was detected by CCK8 assay. d HUVEC migration was assessed by counting the cells on the lower surface of the membrane. Scale bar, $100 \mu \mathrm{m}$. e HUVECs were incubated with CMs from A549 cells in 48-well plates, and their tube formation abilities were examined based on the number of tubes per field. ${ }^{*} p<0.05 ;{ }^{* *} p<0.01$. (TIF $8743 \mathrm{~kb}$ )

Additional file 14: Figure S9. Correlation of EHD1 with $\beta 2 A R, V E G F A$ and CD31 expression and angiogenesis in HCC tissues. a Representative IHC images of NSCLC samples with low and high expression of the indicated proteins. Scale bar, $50 \mu \mathrm{m}$ and $100 \mu \mathrm{m}$. b-d The tissue samples were divided into two groups according to the level of EHD1 expression: the low-expression group (scores of 0 and 1) and the high-expression group (scores of 2 and 3). Patients with high EHD1 expression showed high $\beta 2 A R$ expression (b), VEGFA expression (c), CD31 (d) and MVD (e). e The horizontal lines indicate the intermediate values; the bottom and top of the box represent the 25 th and 75 th percentiles, respectively; and vertical bars represent the data range. (TIF $9217 \mathrm{~kb}$ )

\section{Abbreviations}

CM: conditioned medium; Co-IP: coimmunoprecipitation (Co-IP); EHD1: Mammalian Eps15 homology domain 1; ELISA: enzyme-linked immunosorbent assay (ELISA); HUVEC: Human umbilical vein endothelial cell; ICl: ICl118,551; IF: immunofluorescence (IF); IHC: immunohistochemistry (IHC); ISO: isoproterenol; NSCLC: non-small cell lung cancer; OD: optical density; OS: overall survival; qRT-PCR: quantitative real-time reverse transcriptionpolymerase chain reaction; TCGA: The Cancer Genome Atlas; VEGFA: vascular endothelial growth factors $A$; $\beta 2 A R$ : $\beta 2$-adrenoceptors

\section{Acknowledgements}

Not applicable.

\section{Funding}

This work was supported in part by grants from Certificate of China Postdoctoral Science Foundation Grant (2017 M621307), Hei Long Jiang Postdoctoral Foundation, Hai Yan Youth fund (JJQN2018-11, JJQN2018-02) and the NSFC (Grant Nos. 81772474, 81572276, 81803023, 81672931 and 81602717).

\section{Availability of data and materials}

All data generated or analysed during this study are included in this published article [and its Additional files].

\section{Authors' contributions}

$C L, X Y$ and $W T$ designed this research. $X Y$ and $W T$ carried out most of the experiments, analyzed the data, drew the figures and drafted this manuscript. WT, XY, MQW and LHL helped with cell culture, western blot experiments, GRT-PCR, IP and the CCK-8 assay. LW, XXY, HJ and CY helped with the IHC assay. YS, SY and XXY helped with the animal experiment. HJ, $C Y$ and JDX helped check the manuscript and figures. All authors read and approved the final manuscript.

\section{Ethics approval}

NSCLC tissues were taken from patients undergoing surgical resection at the Harbin Medical University Cancer Hospital (Harbin, China). All work was approved by the Ethics Committee of Harbin Medical University. All animal work was performed in compliance with the Institutional Animal Care and Use Committee (IACUC) of the second affiliated hospital of Harbin medical university in China and the NIH Guide for the Care and Use of Laboratory Animals.

\section{Consent for publication}

We have obtained consents to publish this paper from all the participants of this study.

\section{Competing interests}

The authors declare that they have no competing interests. 


\section{Publisher's Note}

Springer Nature remains neutral with regard to jurisdictional claims in published maps and institutional affiliations.

\section{Author details}

${ }^{1}$ The Fourth Department of Medical Oncology, Harbin Medical University Cancer Hospital, 150 Haping Road, Harbin 150040, China. ${ }^{2}$ The Sixth Department of Medical Oncology, Harbin Medical University Cancer Hospital, 150 Haping Road, Harbin 150040, China. ${ }^{3}$ Department of Orthopedic Surgery, The Second Affiliated Hospital of Harbin Medical University, Xuefu Road 246, Harbin 150081, China.

Received: 17 February 2019 Accepted: 1 April 2019 Published online: 25 April 2019

\section{References}

1. Hirsch FR, Scagliotti GV, Mulshine JL, Kwon R, Curran WJ Jr, Wu YL, et al. Lung cancer: current therapies and new targeted treatments. Lancet. 2017 389:299-311.

2. Miller $K D$, Siegel $R L$, Lin $C C$, Mariotto $A B$, Kramer $J$, Rowland $J H$, et al Cancer treatment and survivorship statistics, 2016. CA Cancer J Clin. 2016;66:271-89.

3. Altorki NK, Markowitz GJ, Gao D, Port JL, Saxena A, Stiles B, et al. The lung microenvironment: an important regulator of tumour growth and metastasis. Nat Rev Cancer. 2019;19:9-31.

4. Ge G, Wang A, Yang J, Chen Y, Yang J, Li Y, et al. Interleukin-37 suppresses tumor growth through inhibition of angiogenesis in non-small cell lung cancer. Journal of experimental \& clinical cancer research : CR. 2016;35:13.

5. Naslavsky N, Boehm M, Backlund PS Jr, Caplan S. Rabenosyn-5 and EHD1 interact and sequentially regulate protein recycling to the plasma membrane. Mol Biol Cell. 2004;15:2410-22.

6. Galperin E, Benjamin S, Rapaport D, Rotem-Yehudar R, Tolchinsky S, Horowitz M. EHD3: a protein that resides in recycling tubular and vesicular membrane structures and interacts with EHD1. Traffic. 2002;3:575-89.

7. Lee DW, Zhao X, Scarselletta S, Schweinsberg PJ, Eisenberg E, Grant BD, et al. ATP binding regulates oligomerization and endosome association of RME-1 family proteins. J Biol Chem. 2005;280:17213-20.

8. Tong D, Liang YN, Stepanova AA, Liu Y, Li X, Wang L et al. Increased Eps15 homology domain 1 and RAB11FIP3 expression regulate breast cancer progression via promoting epithelial growth factor receptor recycling. Tumour Biol 2017:39:1010428317691010.

9. Rotem-Yehudar R, Galperin E, Horowitz M. Association of insulin-like growth factor 1 receptor with EHD1 and SNAP29. J Biol Chem. 2001:276:33054-60.

10. Cypher LR, Bielecki TA, Adepegba O, Huang L, An W, Iseka F, et al. CSF-1 receptor signalling is governed by pre-requisite EHD1 mediated receptor display on the macrophage cell surface. Cell Signal. 2016;28:1325-35.

11. Liu Y, Liang Y, Li M, Liu D, Tang J, Yang W, et al. Eps15 homology domain 1 promotes the evolution of papillary thyroid cancer by regulating endocytotic recycling of epidermal growth factor receptor. Oncol Lett. 2018; 16:4263-70

12. Chan JK, Kiet TK, Blansit K, Ramasubbaiah R, Hilton JF, Kapp DS, et al. MiR378 as a biomarker for response to anti-angiogenic treatment in ovarian cancer. Gynecol Oncol. 2014;133:568-74.

13. Meng $Q$, Xing $Y$, Ren $T$, Lu H, Xi Y, Jiang $Z$, et al. Mammalian Eps15 homology domain 1 promotes metastasis in non-small cell lung cancer by inducing epithelial-mesenchymal transition. Oncotarget. 2017:8:22433-42.

14. Wang $X$, Yin $H$, Zhang H, Hu J, Lu H, Li C, et al. NF-kappaB-driven improvement of EHD1 contributes to erlotinib resistance in EGFR-mutant lung cancers. Cell Death Dis. 2018;9:418.

15. Gao J, Meng Q, Zhao Y, Chen X, Cai L. EHD1 confers resistance to cisplatin in non-small cell lung cancer by regulating intracellular cisplatin concentrations. BMC Cancer. 2016;16:470.

16. Bang I, Choi HJ. Structural features of beta2 adrenergic receptor: crystal structures and beyond. Mol Cells. 2015;38:105-11.

17. Fan X, Gu X, Zhao R, Zheng Q, Li L, Yang W et al. Cardiac beta2-Adrenergic Receptor Phosphorylation at Ser355/356 Regulates Receptor Internalization and Functional Resensitization. 2016;11:e0161373.

18. Huang Q, Tan Q, Mao K, Yang G, Ma G, Luo P, et al. The role of adrenergic receptors in lung cancer. Am J Cancer Res. 2018;8:2227-37.
19. Garg J, Feng YX, Jansen SR, Friedrich J, Lezoualc'h F, Schmidt M, et al. Catecholamines facilitate VEGF-dependent angiogenesis via beta2adrenoceptor-induced Epac1 and PKA activation. Oncotarget. 2017;8:44732-48.

20. Mulcrone PL, Campbell JP, Clement-Demange L, Anbinder AL, Merkel AR, Brekken RA, et al. Skeletal colonization by breast Cancer cells is stimulated by an osteoblast and beta2AR-dependent neo-Angiogenic switch. J Bone Miner Res. 2017:32:1442-54.

21. Folkman J. Angiogenesis: an organizing principle for drug discovery? Nat Rev Drug Discov. 2007;6:273-86.

22. Lu Y, Xu Q, Zuo Y, Liu L, Liu S, Chen L, et al. Isoprenaline/beta2-AR activates Plexin-A1/NEGFR2 signals via VEGF secretion in gastric cancer cells to promote tumor angiogenesis. BMC Cancer. 2017;17:875.

23. Cai H, Liu X, Zheng J, Xue Y, Ma J, Li Z, et al. Long non-coding RNA taurine upregulated 1 enhances tumor-induced angiogenesis through inhibiting microRNA-299 in human glioblastoma. Oncogene. 2017:36:318-31.

24. Zhang JX, Chen ZH, Chen DL, Tian XP, Wang CY, Zhou ZW, et al. LINC01410-miR-532-NCF2-NF-kB feedback loop promotes gastric cancer angiogenesis and metastasis. Oncogene. 2018;37:2660-75.

25. Xing Y, Liu Y, Liu T, Meng Q, Lu H, Liu W, et al. TNFAIP8 promotes the proliferation and cisplatin chemoresistance of non-small cell lung cancer through MDM2/p53 pathway. Cell Commun Signal. 2018;16:43.

26. Tang Z, Li C, Kang B, Gao G, Li C, Zhang Z. GEPIA: a web server for cancer and normal gene expression profiling and interactive analyses. Nucleic Acids Res. 2017:45:W98-w102.

27. Karaman S, Leppanen VM, Alitalo K. Vascular endothelial growth factor signaling in development and disease. Development. 2018;145.

28. Simons M, Gordon E, Claesson-Welsh L. Mechanisms and regulation of endothelial VEGF receptor signalling. Nat Rev Mol Cell Biol. 2016;17:611-25.

29. Li X, DiFiglia M. The recycling endosome and its role in neurological disorders. Prog Neurobiol. 2012;97:127-41.

30. Irannejad R, von Zastrow M. GPCR signaling along the endocytic pathway. Curr Opin Cell Biol. 2014;27:109-16.

31. Mitrofanova I, Zavyalova M, Riabov V, Cherdyntseva N, Kzhyshkowska J. The effect of neoadjuvant chemotherapy on the correlation of tumor-associated macrophages with CD31 and LYVE-1. Immunobiology. 2018:223:449-59.

32. Rofstad EK, Huang R, Galappathi K, Andersen LM, Wegner CS, Hauge A, et al. Functional intratumoral lymphatics in patient-derived xenograft models of squamous cell carcinoma of the uterine cervix: implications for lymph node metastasis. Oncotarget. 2016;7:56986-97.

33. Sandler A, Gray R, Perry MC, Brahmer J, Schiller JH, Dowlati A, et al. Paclitaxel-carboplatin alone or with bevacizumab for non-small-cell lung cancer. N Engl J Med. 2006;355:2542-50.

34. Eswarappa SM, Fox PL. Antiangiogenic VEGF-ax: a new participant in tumor angiogenesis. Cancer Res. 2015:75:2765-9.

35. Ding C, Luo J, Fan X, Li L, Li S, Wen K, et al. Elevated Gab2 induces tumor growth and angiogenesis in colorectal cancer through upregulating VEGF levels. Journal of experimental \& clinical cancer research : CR. 2017;36:56.

36. Hanyaloglu AC, von Zastrow M. Regulation of GPCRs by endocytic membrane trafficking and its potential implications. Annu Rev Pharmacol Toxicol. 2008:48:537-68.

37. Schonegge AM, Gallion J, Picard LP, Wilkins AD, Le Gouill C, Audet M, et al. Evolutionary action and structural basis of the allosteric switch controlling beta2AR functional selectivity. Nat Commun. 2017:8:2169.

Ready to submit your research? Choose BMC and benefit from:

- fast, convenient online submission

- thorough peer review by experienced researchers in your field

- rapid publication on acceptance

- support for research data, including large and complex data types

- gold Open Access which fosters wider collaboration and increased citations

- maximum visibility for your research: over $100 \mathrm{M}$ website views per year

At $\mathrm{BMC}$, research is always in progress.

Learn more biomedcentral.com/submission 